\title{
Efecto del contenido de oxígeno y metano en la cinética de oxidación de un carbonizado de carbón
}

\author{
Jennifer Laverde- Múnera ${ }^{1}$, Pedro Nel Alvarado-Torres², Wilson Ruiz- Machado,* \\ ${ }^{1}$ Grupo de Química de Recursos Energéticos y Medio Ambiente, Instituto de Química, \\ Facultad de Ciencias Exactas y Naturales, Universidad de Antioquia, Medellín, Colombia \\ ${ }^{2}$ Materiales Avanzados y Energía (MATYER), Facultad de Ingenierías, \\ Instituto Tecnológico Metropolitano (ITM), Medellín, Colombia \\ ${ }^{3}$ Grupo de Química de Recursos Energéticos y Medio Ambiente, Instituto de Química, \\ Facultad de Ciencias Exactas y Naturales, Universidad de Antioquia, Medellín, Colombia
}

\begin{abstract}
Resumen
Se evaluó el efecto de la adición de metano en un carbonizado de carbón sometido a reacción con concentraciones de oxígeno bajas. Desde un punto de vista cinético, dicho efecto es determinando por la energía de activación (Ea) y por el factor preexponencial para diferentes concentraciones de oxígeno y mezclas de oxígeno y metano. Se preparó un carbonizado con un tamaño de partícula de 75 a $150 \mu \mathrm{m}$ a partir de un carbón bituminoso altamente volátil, y se determinaron los parámetros cinéticos de la oxidación empleando el método termogravimétrico de velocidad máxima. Las variables evaluadas en la cinética de oxidación fueron la concentración de oxígeno (5, 8 y 21 \%), y el efecto de la adición de metano en el medio de reacción (5, 10 y 14 \% de metano mezclado con 5 \% de oxígeno). Los resultados mostraron que la Ea disminuía al aumentar la concentración de oxígeno, en tanto que al agregar metano se observó un efecto promotor cuando este correspondía al $5 \%$ de la mezcla total; sin embargo, cuando el porcentaje de metano aumentaba, la Ea también lo hacía debido a la rápida reacción del oxígeno con el metano en fase homogénea comparada con la reacción heterogénea del oxígeno con el carbonizado. La Ea obtenida con 5 \% de oxígeno fue de 122,9 kJ/mol y con $21 \%$, de 90,69 kJ/mol; en presencia de metano al $5 \%$ y 5 \% de oxígeno, la Ea fue de 110,7 kJ/mol, y aumentó hasta 170,8 kJ/mol cuando el metano representaba el 14 \% de la mezcla. @ 2017. Acad. Colomb. Cienc. Ex. Fis. Nat.

Palabras clave: Parámetros cinéticos; Carbonizado de carbón pulverizado; Contenido de metano; Contenido de oxígeno.
\end{abstract}

Effect of oxygen and methane on the oxidation kinetics of coal char

\begin{abstract}
In this work we evaluated the effect of adding methane during the oxidation process of a coal char with low oxygen concentration. We analyzed the effect from a kinetic point of view and we determined the activation energy (Ea) and the pre-exponential factor (A) with different oxygen concentrations and oxygen and methane mixtures. We used a coal char from a highly volatile bituminous coal with particle size of 75 to $150 \mu \mathrm{m}$; the kinetic parameters were determined by thermogravimetric method (TGA) with the high-velocity model; oxygen concentration variations were studied with 5,8 and $21 \%$ of oxygen, as well as the effect of adding methane $(5,10$ and $14 \%)$ when oxidation occurred with $5 \%$ of oxygen. The results showed that the Ea decreased when oxygen concentration increased, and that adding $5 \%$ of methane to the total mixture enhanced the effect. Instead, when we increased the methane percentage, the Ea increased due to the rapid reaction of oxygen and methane in the homogeneous phase as compared with the heterogenous reaction of oxygen and coal char. With $5 \%$ oxygen the Ea value was $122.9 \mathrm{~kJ} / \mathrm{mol}$ and with $21 \%$, it was $90.69 \mathrm{~kJ} / \mathrm{mol}$; when methane was added at 5\%, the Ea value was $110.7 \mathrm{~kJ} / \mathrm{mol}$, and it increased to 170.8 $\mathrm{kJ} / \mathrm{mol}$ when there was $14 \%$ of methane in the mixture. (C) 2017. Acad. Colomb. Cienc. Ex. Fis. Nat.
\end{abstract}

Key words: Kinetic parameters; Pulverized coal char; Methane content; Oxygen content.

\section{Introducción}

A través de los años el carbón ha sido un recurso de vital importancia ya que a partir de éste se obtiene energía por medio del proceso de combustión, allí se encuentran involucradas diferentes etapas que pueden ocurrir de manera consecutiva o simultáneamente y cuando se realiza de manera convencional genera además del $\mathrm{CO}_{2}$ grandes emisiones de contaminantes a la atmósfera de especies tales como NOx y SOx. Las emisiones de óxidos de nitrógeno dependen del tipo de tecnología empleada, existiendo numerosas investigaciones

\footnotetext{
*Correspondencia:

Wilson Ruiz- Machado,wilson.ruiz@udea.edu.com

Recibido: 07 de febrero de 2017

Aceptado: 01 de abril de 2017
} 
que apuntan a la reducción de éstas de acuerdo a la variación de parámetros de operación, como por ejemplo, la tecnología de captura de $\mathrm{CO}_{2}$ conocida como oxicombustión en la que se emplea oxígeno puro obtenido de la separación del nitrógeno del aire disminuyendo drásticamente las emisiones de los NOx por la ausencia de nitrógeno (Perrin, et al., 2014). Existen además otro tipo de tecnologías, las cuales emplean para la reducción de contaminantes técnicas de "reburning", donde en una de las etapas se inyecta combustible para crear una zona de atmósfera reductora y así reducir las especies de $\mathrm{NO}$ a $\mathrm{N}_{2}$, mientras que en una etapa posterior se inyecta sólo aire para obtener un exceso de éste y quemar el combustible del "reburning" (Shen, Liu, Ma, Zhang, \& Jiang, 2015). Actualmente se están adelantando estudios sobre una nueva metodología de combustión que se caracteriza por una combustión volumétrica, éste concepto fue propuesto inicialmente en el año 2008 caracterizándose por un mezclado intenso de los gases y una alta recirculación de ellos dentro de las cámaras de combustión, por tanto, la combustion se presenta en una zona extensa evitando la formación de pequeños lugares de reacción ya que hay una mejor distribución de las especies reactantes, los flujos y las temperaturas (J. Li, Yang, Blasiak, \& Ponzio, 2012); a éste tipo de proceso de combustión también se le conoce como combustión sin llama o MILD combustion (por sus siglas, moderate or intense low-oxygen dilution) y puede ser aplicada tanto para carbón como para metano; gracias a sus características se logra una baja concentración de oxígeno al interior del horno (menor a 8\%) debido a la alta recirculación de los gases que lo diluyen y se genera una gran disminución en las emisiones de NO (menores a 400 ppm, valores obtenidos para la combustión sin llama del carbón comparativamente con valores de 800-1000 ppm obtenidos para combustión convencional) (Cavaliere, \& De Joannon, 2004) (Weber, Smart, \& Vd Kamp, 2005). También existen trabajos que se enfocan principalmente en dar explicación a las rutas de formación de los óxidos de nitrógeno bajo éste régimen cuando el combustible empleado es el metano como el presentado por (Alvarado, Cadavid, Mondragón, Ruiz, \& Amell, 2009), o estudios que evalúan parámetros de variación de diseño del quemador que opera bajo éste régimen como por ejemplo, la posición de inyectores para mejorar el sistema de recirculación de los gases, haciendo que las emisiones de NO sean reducidas incluso hasta valores tan bajos como 200 ppm aproximadamente (Bilgen, 2014). Sin embargo, a pesar de ofrecer la gran ventaja de disminuir las emisiones de $\mathrm{NOx}$ y $\mathrm{CO}_{2}$, se ha demostrado en recientes estudios que la técnica tiene como desventaja la generación de cantidades considerables de inquemados sólidos al emplearse para la combustión de carbón disminuyendo la eficiencia del proceso, en la tesis doctoral de (Torres Alvarado, 2014), se concluye que cuando hay baja concentración de oxígeno durante la combustión de un material carbonoso, la reactividad disminuye conforme incrementa la conversión y lo asocian a la disminución de especies reactivas de tipo $\mathrm{sp}^{3}$ de estructura amorfa que conlleva al aumento del grado de ordenamiento del material haciendo que este sea menos reactivo. Otros trabajos evalúan el efecto del tamaño de partícula para un carbón de bajo rango, se ve una dependencia del tamaño con el porcentaje de consumo del mismo, donde a mayores tamaños de partícula, el consumo total de carbono es menor, esto es debido a que el tamaño de partícula se asocia con el tiempo de residencia ya que partículas más grandes son más pesadas que las de menor tamaño requiriendo entonces mayor tiempo de residencia para lograr su consumo (Medwell \& Chinnici, 2017).

Con el fin de lograr una disminución de los inquemados sólidos debidos a la baja concentración de oxígeno, se ha planteado la co-combustión de carbón pulverizado con metano bajo régimen de combustión sin llama o MILD combustion como estrategia útil, ya que la adición de metano podría tener un efecto químico en cuanto a incrementar la cantidad de radicales que pueden mejorar la reactividad del material carbonoso gracias a la promoción de especies radicalarias como $* \mathrm{OH}$ que son útiles durante el proceso de oxidación especialmente en la etapa de terminación (Liotta, R., Brons, G., Isaacs, 1983). Sin embargo, existe una limitante en cuanto a la poca información sobre estudios que ayuden a explicar el efecto que tendría el metano durante la oxidación del carbonizado de carbón. Por lo tanto para tener una aproximación al proceso de co-combustión y entenderlo, es importante comenzar a realizar estudios fundamentales del fenómeno tales como la determinación de los parámetros cinéticos para la oxidación heterogénea del carbonizado cuando en la atmósfera de reacción se incluye el metano, ya que la obtención de parámetros cinéticos se puede relacionar con condiciones de diseño de cámaras de combustión que operen bajo este régimen; la técnica termogravimétrica ha sido de gran utilidad para desarrollar métodos de obtención de estos parámetros, siendo sencilla y de bajo costo (Shi, Liu, Guo, He, \& Liu, 2014); para el caso de la oxidación se encuentra gran cantidad de reportes donde la técnica es empleada para determinar modelos cinéticos de oxidación a temperaturas altas y bajas mostrando resultados favorables y reproducibles (Jones, Chiz, Koh, \& Matthew, 1996).

El objetivo de este trabajo es evaluar el efecto de la relación $\mathrm{O}_{2} / \mathrm{CH}_{4}$ en la cinética de la reacción con baja concentración de oxígeno de un carbonizado de carbón pulverizado, empleando la técnica termogravimétrica no-isotérmica como herramienta para la obtención de los parámetros cinéticos.

\section{Metodología}

Preparación del material carbonoso. El material seleccionado fue un carbón Colombiano tipo bituminoso alto en volátiles proveniente de la región de Amagá-Antioquia, cuyo poder calorífico superior es de 24,33 MJ/Kg (medido en bomba calorimétrica), este tipo de carbón se selecciona debido a que existen estudios que demuestran que para carbones de alto rango la cantidad de inquemados sólidos es 
mayor comparativamente con carbones de menor rango, en un estudio realizado por investigadores del Centre for Energy Technology de la universidad de Adelaida en Australia, se obtuvo un porcentaje de inquemados cercano al 10\% para un carbón de rango bajo, mientras que el porcentaje de inquemados para un carbón de alto rango tipo antracita fue de alrededor del 60\% (Saha, Chinnici, Dally, \& Medwell, 2015). El carbón fue pulverizado en un molino de bolas y tamizado con mallas 200-100 que según la norma ASTM (United \& Of, n.d.) corresponden a un tamaño de partícula entre 75-150 $\mu \mathrm{m}$. El carbonizado se obtuvo por medio de proceso de pirólisis en un horno horizontal como empleando atmósfera de nitrógeno con flujo total de $100 \mathrm{~mL} / \mathrm{min}$, empleando una velocidad de calentamiento de $20^{\circ} \mathrm{C} / \mathrm{min}$ hasta una temperatura final de $950^{\circ} \mathrm{C}$, donde se mantuvo en isoterma por una hora. Posteriormente la muestra se enfrió hasta temperatura ambiente y se guardó el carbonizado obtenido en un desecador para evitar adsorción de humedad. El análisis próximo y elemental tanto para el carbón fresco como el carbonizado se presenta en Tabla 1.

De acuerdo a los porcentajes mostrados en la Tabla 1, el carbón es clasificado como bituminoso-alto en volátiles, con un poder calorífico superior de $23,96 \mathrm{MJ} / \mathrm{kg}$ calculado de acuerdo a la ecuación propuesta por Lloyd-Davenport (Friedl, Padouvas, Rotter, \& Varmuza, 2005) tomando en cuenta el análisis último, resultado que tiene concordancia con el obtenido por medio de la medida obtenida en la bomba calorimétrica cuyo valor fue de 24,33 MJ/kg y que es correspondiente a éste tipo de carbones, afianzando así los resultados de caracterización. La ecuación 1 muestra la aproximación de Lloyd-D:

$$
\operatorname{HHV}\left(\frac{k J}{k g}\right)=357.77 C+917.58 H-84.51 O-59.38 N+111.87 S(1)
$$

Cinética de la oxidación del carbonizado con diferentes concentraciones de oxígeno y mezclas de oxígeno/metano. Los experimentos para determinar los parámetros cinéticos se llevaron a cabo en un analizador termogravimétrico de la serie SDT Q-600 de TA Instruments. Para las reacciones se empleó un tamaño de muestra de 10-20 mg de carbonizado sobre crisoles de óxido de aluminio $\left(\mathrm{Al}_{2} \mathrm{O}_{3}\right)$ y la energía de activación (Ea) y el factor pre-exponencial (A) fueron obtenidos empleando el método de la velocidad máxima (Acelas, 2010) donde las velocidades de calentamiento empleadas fueron $(3,5,10,15 \text { y } 20)^{\circ} \mathrm{C} / \mathrm{min}$. Se evaluaron 3 concentraciones de oxígeno: 5,8 y 21\%, dos correspondientes a atmósfera diluida (5\% y $8 \%$ ) y una correspondiente a atmósfera convencional
(21\%). Estas concentraciones se lograron al mezclar oxígeno puro con argón para un flujo total de $100 \mathrm{~mL} / \mathrm{min}$ empleando controladores de flujo másico marca Brooks 5850 e. Con el fin de evaluar el efecto del metano en la cinética de la oxidación del carbonizado bajo régimen de combustión sin llama o MILD combustion, se realizaron mezclas de metano-oxígeno, manteniendo 5\% de oxígeno (concentración típicamente obtenida bajo este régimen de combustión) y variando el metano a 5\%, 10\% y 14\% en la mezcla gaseosa total. Las muestras fueron calentadas desde temperatura ambiente hasta una temperatura final de $850^{\circ} \mathrm{C}$ y mantenidas en isoterma por 30 minutos.

Modelo matemático empleado para la determinación de parámetros cinéticos

Método de la velocidad máxima. Para las reacciones heterogéneas como la oxidación del carbón, la cinética se puede determinar por el cambio de las propiedades del material sólido con el tiempo, ésta reacción está gobernada por la ley de primer orden de Arrhenius (Gil, Casal, Pevida, Pis, \& Rubiera, 2010), así la cinética de la reacción vendrá dada por la ecuación 2

$$
\frac{d x}{d t}=A \exp \left(\frac{-E}{R T}\right) * f(x) ; x=\left(\frac{m_{0}-m_{t}}{m_{0}-m_{\infty}}\right)
$$

En esta ecuación x representa la conversión, m la masa, t el tiempo y $f(x)$ es el modelo de mecanismo de reacción de la partícula. Por principio matemático (Masel, 1996), la velocidad máxima del proceso de oxidación puede ser obtenida de acuerdo a la ecuación 3 así:

$$
\left(\frac{d X^{2}}{d T^{2}}\right)=0
$$

La descomposición de un sólido desde un punto cinético es usualmente descrita como:

$$
\begin{aligned}
\left(\frac{d X}{d t}\right)=A e^{-\left(\frac{E_{a}}{R T}\right)} & (1-x)^{n} ; \text { con } \\
& n=\text { orden de reacción, } \\
& R=\text { constante de los gases, } \\
& A=\text { factor pre-exponencial y } \\
& \text { Ea = energía de activación }
\end{aligned}
$$

Expresando entonces la ecuación cinética 4 en función de la velocidad de calentamiento:

$$
\left(\frac{d X}{d t}\right)=\left(\beta \frac{d X}{d T}\right)=A e^{-\left(\frac{E_{a}}{R T}\right)}(1-x)^{n}
$$

La ecuación 3 puede ser expresada como:

$$
\left(\frac{d X^{2}}{d T^{2}}\right)=\frac{d\left(-\beta \frac{d X}{d T}\right)}{d T}=0
$$

Tabla 1. Análisis próximo y último del material carbonoso.

\begin{tabular}{ccccccccc}
\hline \multirow{2}{*}{ Muestra } & \multicolumn{2}{c}{ *Análisis Próximo } & \multicolumn{3}{c}{ Análisis Elemental } \\
\cline { 2 - 8 } & Volátiles & Carbono Fijo & Cenizas & $\boldsymbol{C}$ & $\boldsymbol{H}$ & $\boldsymbol{N}$ & $* * \boldsymbol{O}$ & $\boldsymbol{S}$ \\
\hline Carbón fresco & 42,80 & 51,30 & 5,92 & 61,30 & 4,66 & 1,26 & 26,30 & 0,45 \\
Carbonizado & 7,140 & 84,70 & 8,13 & 72,10 & 1,46 & 0,90 & 16,80 & 0,59 \\
\hline
\end{tabular}

*Sobre base seca, **Porcentaje obtenido por diferencia 
Si se reemplaza la ecuación 5 en la ecuación 6 y se resuelve la derivada se obtiene:

$$
\ln \left[\frac{\beta^{2}}{T_{m}^{2}\left(1-X_{m}\right)^{n-1}}\right]=\ln \frac{n A R}{E_{a}}-\frac{E_{a}}{R T_{m}}
$$

Siendo $\mathrm{T}_{\mathrm{m}}$ la temperatura a la velocidad máxima de reacción, en este caso la reacción de oxidación, y $\mathrm{X}_{\mathrm{m}}$ es la conversión a la velocidad máxima de reacción, teniendo en cuenta como orden de reacción para el carbonizado con el oxígeno como $n=1$ (Wang, Zhang, Shao, Sun, \& Zuo, 2014), la ecuación 7 se transforma en:

$$
\ln \frac{\beta}{T_{m}^{2}}=\ln \frac{A R}{E_{a}}-\frac{E_{a}}{R T_{m}}
$$

Para obtener los parámetros cinéticos a partir de la ecuación 8 se necesita determinar experimentalmente la temperatura a la velocidad máxima de reacción que es obtenida a partir de las curvas DTG a diferentes velocidades de calentamiento, ésta temperatura es necesaria para graficar $\ln \left(\beta / \mathrm{T}_{\mathrm{m}}{ }^{2}\right)$ vs $1 / \mathrm{Tm}$ y así determinar los parámetros cinéticos deseados a partir de la pendiente y el intercepto obtenidos de la regresión lineal (S. Li, \& Yue, 2003); en este artículo se hablará de éste modelo como velocidad máxima A.

Gracias a las diferentes velocidades de calentamiento empleadas experimentalmente, también es posible obtener los valores de conversión máxima a una temperatura máxima $\left(T_{m}\right)$ de reacción, estas conversiones se pueden emplear de igual manera en la determinación de los parámetros cinéticos al aproximar la ecuación 8 de la siguiente manera:

$$
\ln \left[\frac{\left(1-\ln \left(1-X_{\text {max }}\right)\right) \beta}{T_{m}^{2}}\right] \approx \ln \frac{A R}{E_{a}}-\frac{E_{a}}{R T_{m}}
$$

Es así como, los parámetros cinéticos son calculados a partir de la pendiente e intercepto de la línea recta obtenida al graficar $\ln \left[\frac{\left(1-\ln \left(1-X_{m i x}\right)\right)}{T_{m}^{2}}\right]$ respecto al inverso de la temperatura máxima, en el presente artículo se hablará de éste modelo como velocidad máxima $B$.

$\mathrm{Al}$ emplear diferentes velocidades de calentamientos se obtiene un valor de temperatura máxima asociada a cada velocidad tal como se muestra en la Figura 1, además a cada una de estas temperaturas se asocia de igual manera un valor de conversión máxima, con los datos obtenidos se pueden hallar los parámetros cinéticos para cada atmósfera de reacción de acuerdo a los modelos de regresión lineal antes mencionados.

\section{Resultados}

Cinética de la oxidación del carbonizado con diferentes concentraciones de oxígeno y mezclas de oxígeno/metano

Efecto de la velocidad de calentamiento. Un perfil de combustión típico para los carbonizados de carbón es como el que se presenta en la Figura 2a donde se grafica la pérdida de peso con respecto al tiempo, con una concentración de oxígeno de $8 \%$ y a diferentes velocidades de calentamiento. La primera pérdida que ocurre antes de los 20 min corresponde a la humedad (a una temperatura de $120^{\circ} \mathrm{C}$ aproximadamente), luego se observa una segunda pérdida importante de peso correspondiente a la oxidación de carbonizado la cual comienza entre $350-426{ }^{\circ} \mathrm{C}$, valores típicos reportados en la literatura (Wang, et al., 2014). Cuando se comparan las diferentes velocidades de calentamiento empleadas, se observa una dependencia de la oxidación con ésta, donde la conversión máxima se desplaza a temperaturas mayores implicando tiempos menores cuando la velocidad de calentamiento incrementa, lo que se evidencia en la Figura 2b, afectando la temperatura inicial de pérdida de masa, haciendo que el tiempo de oxidación a altas velocidades de calentamiento sea menor.

Efecto de la concentración de oxígeno. Para evaluar el efecto de la concentración de oxígeno sobre la energía de activación, se hicieron reacciones con concentraciones de $5 \%$, $8 \%$ y $21 \%$ de oxígeno, usando éste último porcentaje como comparativo con atmósfera de combustión convencional; además de determinar el efecto de la concentración de oxígeno, se realizó la oxidación del carbonizado empleando mezclas de 5\% de oxígeno con $(5,10$ y 14)\% de metano para observar el efecto que tiene el metano en el proceso de oxidación; la Figura 3a muestra los perfiles de conversión variando la concentración de oxígeno para la oxidación del carbonizado cuando se mantiene una velocidad de calentamiento de $3^{\circ} \mathrm{C} / \mathrm{min}$ (esta velocidad se toma para comparar el efecto observado a cada concentración de oxígeno a la menor velocidad de calentamiento), se puede observar que a medida que se incrementa la concentración de oxígeno en la atmósfera, la temperatura inicial de reacción no varía significativamente cuando se tiene $5 \%$ y $8 \%$ de oxígeno, siendo esta de aproximadamente $400^{\circ} \mathrm{C}$, sin embargo, cuando se incrementa la concentración de oxígeno a $21 \%$, la temperatura inicial de reacción es de aproximadamente $360^{\circ} \mathrm{C}$, esto debido a la alta disponibilidad de oxígeno en el ambiente de reacción que hace posible que la reacción se lleve a cabo mucho más rápido y consecuentemente a más baja temperatura, lo que se ve reflejado en el tiempo donde

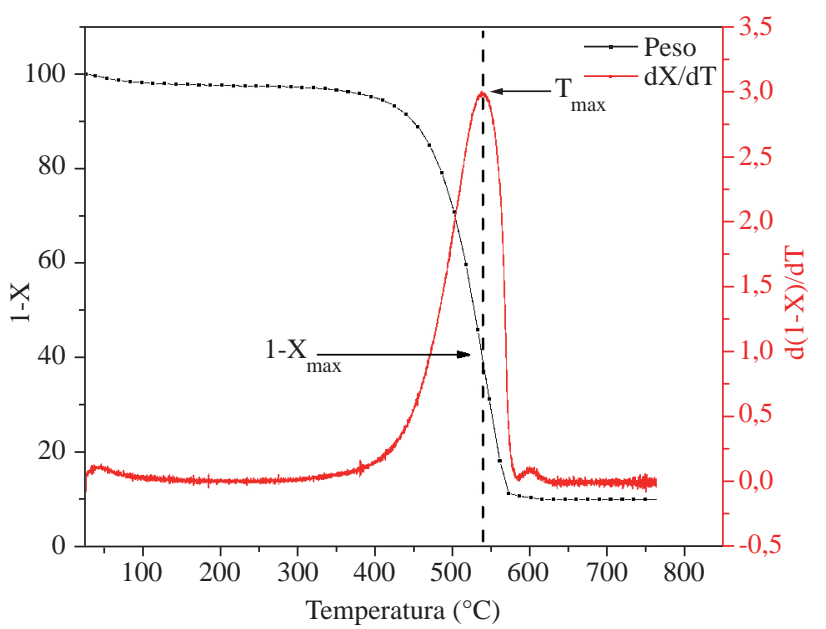

Figura 1. Curvas de TGA y DTG de la oxidación del carbonizado de carbón a $5^{\circ} \mathrm{C} / \mathrm{min}$ para la obtención de los parámetros cinéticos para una atmósfera de 5\% de oxígeno. 

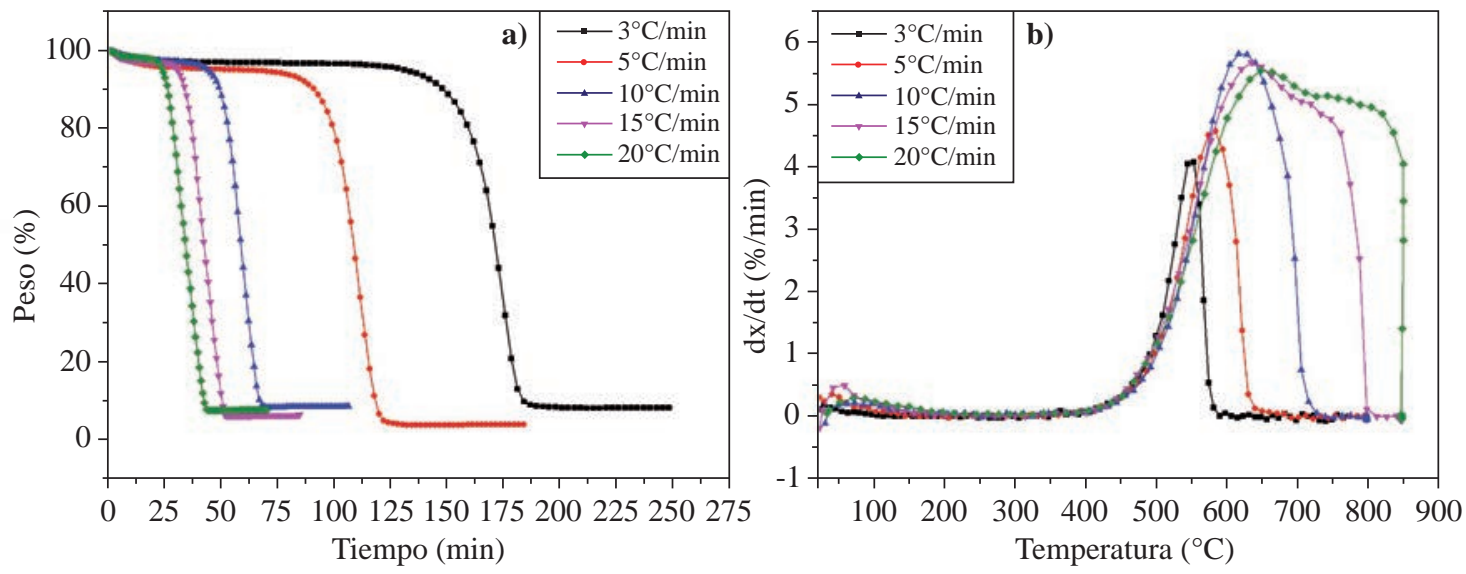

Figura 2. Perfiles de oxidación a diferentes velocidades de calentamiento para una atmósfera compuesta por $8 \%$ de oxígeno.
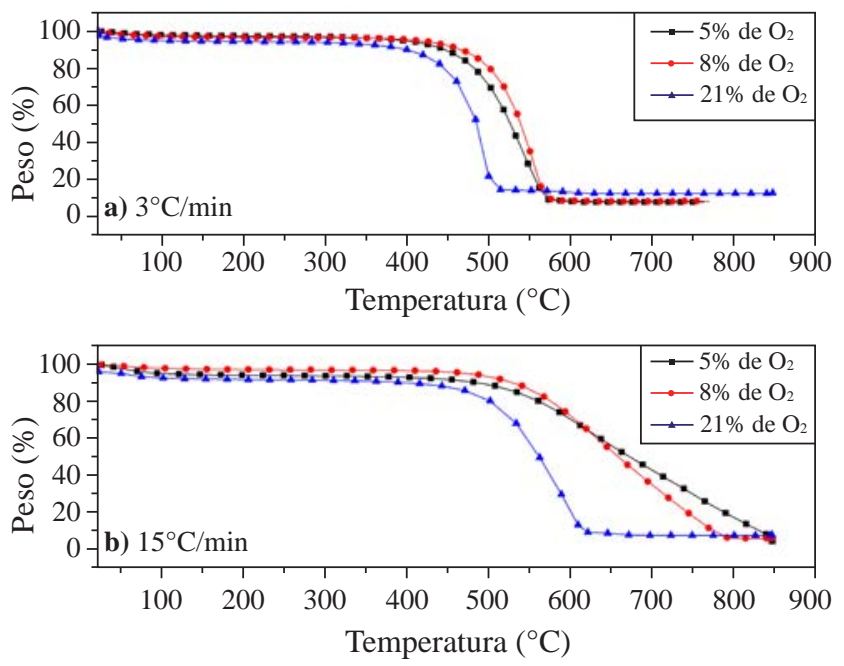

Figura 3. Efecto de la atmósfera en el perfil de oxidación variando la concentración de oxígeno en dos velocidades de calentamiento, a) $3^{\circ} \mathrm{C} / \mathrm{min}$, b) $15^{\circ} \mathrm{C} / \mathrm{min}$.

finaliza la reacción, siendo más corto cuando se emplea 21\% de oxígeno; en la Figura $3 \mathrm{~b}$ se muestran los perfiles de conversión al variar la concentración de oxígeno pero cuando se tiene una velocidad de calentamiento mayor, $15^{\circ} \mathrm{C} / \mathrm{min}$, notándose en este caso que la temperatura inicial de reacción se desplaza a valores más altos que cuando se tiene velocidad de calentamiento más baja, a $500^{\circ} \mathrm{C}$ aproximadamente para $5 \%$ y $8 \%$ de oxígeno y a $450^{\circ} \mathrm{C}$ cuando se tiene $21 \%$ de oxígeno, el comportamiento de los perfiles al inicio es similar al obtenido cuando se calienta a $3^{\circ} \mathrm{C} / \mathrm{min}$, pero al final de la reacción se nota que cuando se emplea $5 \%$ de oxígeno no se alcanza a oxidar completamente el carbonizado ya que no se ve estabilización de la señal de pérdida de peso, mientras que con $8 \%$ y $21 \%$ de oxígeno si, notándose estabilización de la señal cuando llega a los $800^{\circ} \mathrm{C}$ para el caso de $8 \%$ de oxígeno y $620^{\circ} \mathrm{C}$ para el caso de $21 \%$; lo que quiere decir que a bajas velocidades de calentamiento se tiene el tiempo suficiente para que exista la reacción entre el oxígeno y el carbonizado logrando una conversión mayor que cuando se emplean velocidades de calentamiento mayores donde el tiempo de contacto entre el gas y el carbonizado es más corto obteniéndose combustión incompleta.

En la Figura 4a existe ya adición de metano a la atmósfera de reacción y con una velocidad de calentamiento baja; al inicio de la reacción se observa un perfil similar al observado cuando la atmósfera de reacción sólo contiene oxígeno, pero cuando se analiza el tiempo de finalización de la reacción se observa que a medida que se incluye más cantidad de metano al medio, la finalización del consumo de carbonizado ocurre a temperaturas un poco más elevadas $\left(576^{\circ} \mathrm{C}\right.$ cuando solo hay $5 \%$ de oxígeno y $617^{\circ} \mathrm{C}$ cuando en el medio de reacción hay $5 \%$ de oxígeno y $14 \%$ de metano), además el porcentaje de conversión también se ve afectado, disminuyendo cuando se incluye el metano, efecto que se nota más claramente cuando se emplea una velocidad de calentamiento de $15^{\circ} \mathrm{C} /$ min tal y como se observa en la Figura 4b, donde se obtiene
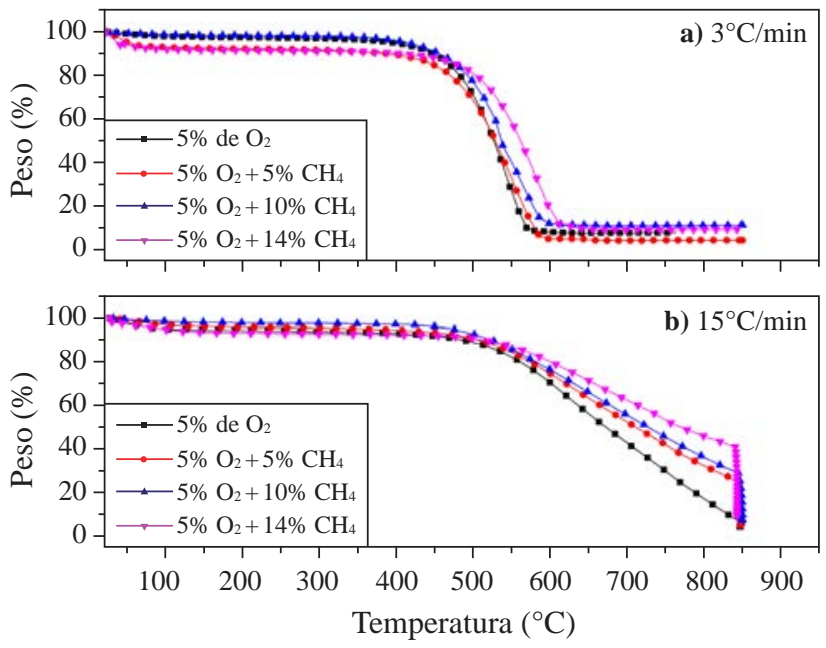

Figura 4. Efecto de la atmósfera en el perfil de oxidación cuando se adiciona metano al medio de reacción en dos velocidades de calentamiento, a) $3^{\circ} \mathrm{C} / \mathrm{min}$, b) $15^{\circ} \mathrm{C} / \mathrm{min}$. 
una conversión de $41 \%$ aproximadamente; esto debido a que ya existe en el sistema una mezcla de dos combustibles en diferente estado de agregación, donde las reacciones en fase homogénea del oxígeno con el metano ocurren de maneras más rápida que las de fase heterogénea, haciendo que se retrase el consumo del carbonizado ya que se debe incluir el efecto de la velocidad de los fenómenos de transferencia entre fases sobre la velocidad propia de la reacción (Izquierdo \& Torres, 2004), efecto que se verá reflejado en los valores de energía de activación obtenidos, siendo en mayor medida cuando se emplea la mezcla de $5 \% \mathrm{O}_{2}$ con $14 \% \mathrm{CH}_{4}$. El comportamiento general con la velocidad de calentamiento es similar al obtenido con atmósfera de oxígeno, donde a velocidades mayores se requiere más tiempo de isoterma para lograr que finalice el consumo del carbonizado.

Las temperaturas de inicio del proceso de oxidación para cada atmósfera estudiada, así como las temperaturas máximas de conversión se encuentran en la Tabla 2, se observa en el caso de las atmósferas compuestas solo por oxígeno, que al aumentar la concentración de este disminuyen las temperaturas de inicio de la oxidación y de conversión máxima, esto debido a la alta disponibilidad de oxígeno que favorece el proceso haciendo que ocurra a menos temperaturas; al incluir metano en el medio de reacción sucede el caso contrario, se observa un aumento de estas cuando el porcentaje de metano en la mezcla aumenta, este comportamiento puede ser debido a las reacciones competitivas de oxidación del metano con respecto a la oxidación del carbonizado de carbón que hacen que el proceso requiera mayor energía para poder que oxide el carbonizado.

Determinación de los parámetros cinéticos. $\mathrm{Al}$ evaluar los modelos matemáticos de velocidad máxima $\boldsymbol{A}$ y $\boldsymbol{B}$ se obtienen como resultado los valores de energía de activación mostrados en la Tabla 3, se puede ver que las energías de activación obtenidas tienen la coherencia esperada de disminuir a medida que aumenta el porcentaje de oxígeno en la mezcla debido a la mayor disponibilidad de éste para reaccionar con el carbonizado de carbón; en cualquiera de los dos casos de modelo evaluados, la energía de activación tiene un valor similar al reportado en la literatura para oxidación con $21 \%$ de oxígeno (se reporta una energía de activación de 93,87 kJ/mol cuando el método no isotérmico se realiza con múltiples velocidades de calentamiento) (Wang, et al., 2014). La Figura 5 muestra como ejemplo la comparación de los datos de regresión lineal cuando se emplean los métodos de velocidad máxima $\boldsymbol{A}$ y $\boldsymbol{B}$ los cuales son empleados para determinar los parámetros cinéticos a una concentración de $5 \%$ de oxígeno.

Para evaluar los parámetros cinéticos de la mezcla de reacción $\mathrm{O}_{2} / \mathrm{CH}_{4}$ con carbonizado de carbón, elegimos el método de velocidad máxima $\boldsymbol{A}$, debido a la concordancia con el valor reportado en la literatura cuando se realiza la oxidación con $21 \%$ de oxígeno a diferentes velocidades de calentamiento y por el valor de $\mathrm{R}^{2}$ que indica mejor correlación en la regresión lineal realizada.
Tabla 2. Temperaturas de conversión para la oxidación con las diferentes atmósferas empleadas.

\begin{tabular}{cccc}
\hline $\begin{array}{c}\text { Velocidad de } \\
\text { calentamiento } \\
\left({ }^{\circ} \mathbf{C} / \text { min) }\right.\end{array}$ & Atmósfera & $\begin{array}{c}\text { Temperatura } \\
\text { de inicio de } \\
\text { la oxidación } \\
\left({ }^{\circ} \mathbf{C}\right)\end{array}$ & $\begin{array}{c}\text { Temperatura } \\
\text { de oxidación } \\
\text { máxima } \\
\left({ }^{\circ} \mathbf{C}\right)\end{array}$ \\
\hline 3 & $5 \% \mathrm{O}_{2}$ & 440,8 & 590,9 \\
& $8 \% \mathrm{O}_{2}$ & 379,7 & 549,5 \\
& $21 \% \mathrm{O}_{2}$ & 282,8 & 490,6 \\
& $5 \% \mathrm{O}_{2} ; 5 \% \mathrm{CH}_{4}$ & 298,8 & 539,9 \\
& $5 \% \mathrm{O}_{2} ; 10 \% \mathrm{CH}_{4}$ & 321,0 & 552,3 \\
& $5 \% \mathrm{O}_{2} ; 14 \% \mathrm{CH}_{4}$ & 392,9 & 579,8 \\
\hline \multirow{2}{*}{5} & $5 \% \mathrm{O}_{2}$ & 468,4 & 674,4 \\
& $8 \% \mathrm{O}_{2}$ & 392,9 & 637,1 \\
& $21 \% \mathrm{O}_{2}$ & 375,1 & 613,1 \\
& $5 \% \mathrm{O}_{2} ; 5 \% \mathrm{CH}_{4}$ & 313,0 & 614,1 \\
& $5 \% \mathrm{O}_{2} ; 10 \% \mathrm{CH}_{4}$ & 336,9 & 625,8 \\
& $5 \% \mathrm{O}_{2} ; 14 \% \mathrm{CH}_{4}$ & 396,5 & 643,3 \\
\hline
\end{tabular}

Tabla 3. Energías de activación obtenidas cuando se emplean las ecuaciones 8 y 9.

\begin{tabular}{ccccc}
\hline $\begin{array}{c}\text { Contenido } \\
\text { de } \mathbf{O}_{2}\end{array}$ & Modelo Cinético & $\begin{array}{c}\text { Energía de } \\
\text { activación } \\
\text { (kJ/mol) }\end{array}$ & $\mathbf{R}^{2}$ \\
\hline $5 \%$ & Velocidad máxima & A & 122,90 & 0,9752 \\
\hline & & B & 164,50 & 0,9697 \\
\hline \multirow{2}{*}{$8 \%$} & Velocidad máxima & A & 102,70 & 0,9787 \\
\cline { 3 - 5 } & & B & 123,20 & 0,9598 \\
\hline \multirow{2}{*}{$21 \%$} & Velocidad máxima & $\mathrm{A}$ & 90,69 & 0,9986 \\
& & $\mathrm{~B}$ & 103,90 & 0,9952 \\
\hline
\end{tabular}

Las energías de activación obtenidas y el factor preexponencial para cada reacción se muestran en la Tabla 4, la disminución de la energía de activación con el aumento del porcentaje de oxígeno sin incluir metano en la mezcla puede ser debido a un mecanismo cinético global el cual es gobernado por la difusión entre el sólido (fase heterogénea) y el gas donde existe mayor cantidad de oxígeno disponible que ayuda a oxidar el material carbonoso. Cuando se incluye metano en la mezcla de reacción con oxígeno al $5 \%$, se observa una disminución de aproximadamente el $10 \%$ en el valor de Ea con respecto al valor obtenido cuando la atmósfera está compuesta únicamente por 5\% de oxígeno, lo que podría estar relacionado con un aumento de la producción de radicales por parte del metano en la fase homogénea tales como $\mathrm{CH}_{3} \mathrm{OO}^{*}$, $\mathrm{HOO}^{*}$ y en mayor proporción los radicales $* \mathrm{OH}$ que es obtenido por abstracción del hidrógeno del metano por compuestos oxidantes. Estas 


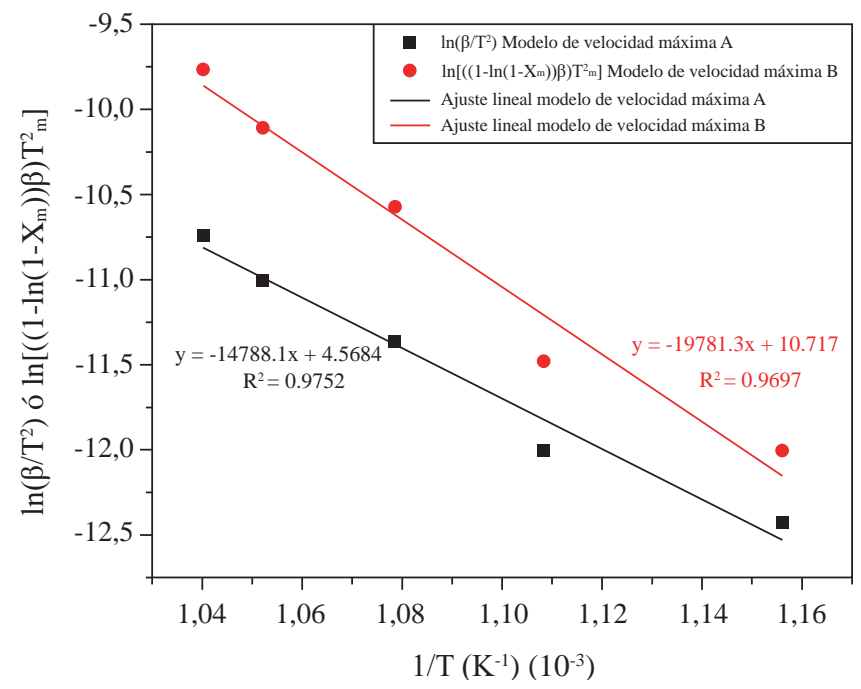

Figura 5. Regresiones lineales para ambos modelos matemáticos empleados en la determinación de los parámetros cinéticos cuando se emplea 5\% de oxígeno como atmósfera de reacción.

Tabla 4. Parámetros cinéticos obtenidos con cada una de las atmósferas empleadas.

\begin{tabular}{cccc}
\hline \multicolumn{4}{c}{ Carbonizado de carbón $(\mathbf{7 5 - 1 5 0 )} \mathbf{\mu m}$} \\
\hline $\begin{array}{c}\text { Oxígeno } \\
\text { \% }\end{array}$ & Metano \% & $\begin{array}{c}\mathbf{E a} \\
(\mathbf{k J} / \mathbf{m o l})\end{array}$ & $\begin{array}{c}\text { A } \\
\left(\mathbf{s}^{-1}\right)\end{array}$ \\
\hline 5 & 0 & 122,90 & 96,39 \\
& 0 (reproducibilidad) & 123,90 & 114,20 \\
\hline & 5 & 110,70 & 54,87 \\
\hline & 10 & 133,80 & $1,130 \times 10^{3}$ \\
\hline 21 & 14 & 170,80 & $1,121 \times 10^{5}$ \\
\hline & 14 (reproducibilidad) & 170,70 & $1,121 \times 10^{5}$ \\
\hline & 0 & 102,70 & 14,00 \\
\hline
\end{tabular}

especies radicalarias pueden generar especies que transfieren hidrógeno, promoviendo el craqueo de los compuestos aromáticos presentes en el carbonizado; esto se asocia a que hay supresión de reacciones secundarias tales como, condensación y re-polimerización (Krerkkaiwan, Fushimi, Tsutsumi, \& Kuchonthara, 2013) (Quan, Xu, An, Liu, 2014) (Park, Kim, Lee, \& Lee, 2010) (Yuan, et al., 2012). Por otro lado, también se puede dar la formación de otros radicales como ${ }^{*} \mathrm{CH} \mathrm{y}{ }^{*} \mathrm{CH}_{3}$, permitiendo entonces una promoción del consumo del carbonizado disminuyendo la energía de activación, algunas reacciones que dan cuenta de esto se muestran a continuación (Alviso, Rolon, Scouflaire, \& Darabiha, 2015):

$$
\begin{array}{ll}
\mathrm{C}_{2} \mathrm{H}+\mathrm{O}_{2} \leftrightarrow \mathrm{CO}+\mathrm{CH}^{*} & \mathrm{RH}+\mathrm{OH}^{*} \leftrightarrow \mathrm{R}^{*} \mathrm{H}_{2} \mathrm{O} \\
\mathrm{C}_{2}+\mathrm{OH} \leftrightarrow \mathrm{CO}+\mathrm{CH}^{*} & \mathrm{R}^{*}+\mathrm{OH}^{*} \leftrightarrow \mathrm{ROH} \\
\mathrm{CH}+\mathrm{O}_{2} \leftrightarrow \mathrm{CO}+\mathrm{OH}^{*} & \mathrm{RCOO}^{*} \rightarrow \mathrm{R}^{*}+\mathrm{CO}_{2}
\end{array}
$$

Los mecanismos más probables de oxidación del metano y otros hidrocarburos implican formación de radicales $\mathrm{H}^{*}$, $\mathrm{O}^{*}$, *OH y HOO*. También se ha reportado que las velocidades de reacción entre especies radicales como $* \mathrm{O}, \mathrm{H}^{*}$ y ${ }^{*} \mathrm{OH}$ con las moléculas de combustible como $\mathrm{CH}_{3} \mathrm{OH}$, $\mathrm{CH}_{4}, \mathrm{C}_{2} \mathrm{H}_{6}$, además de otras, son generalmente mayores que para las reacciones entre estas mismas especies con $\mathrm{CO}$ y $\mathrm{H}_{2}$; por lo tanto, cuando aún permanecen o existen especies de hidrocarburos, la velocidad de reacción de las especies radicalarias $\left(\mathrm{O}^{*}, \mathrm{H}^{*} \mathrm{y} * \mathrm{OH}\right)$ en la oxidación del $\mathrm{CO}$ y el $\mathrm{H}_{2}$ disminuye. Como resultado, no existirá forma alguna para disminuir la concentración de radicales en regiones en las que aún hay cantidades de combustible (Westbrook \& Dryer, 1981) (Tjatjopoulos \& Vasalos, 1992); esto implicaría que al tener metano en la combustión de carbón con bajo contenidos de oxígeno tendría un efecto en cuanto a la formación de radicales $\left(\mathrm{O}^{*}, \mathrm{H}^{*} \mathrm{y} * \mathrm{OH}\right)$, los cuales estarían reaccionando con el material carbonoso por reacciones de abstracción de $\mathrm{H}$ y escisión de enlaces C-C; este conjunto de reacciones modificaría la superficie del material alterando sus propiedades estructurales, la reactividad y la cinética de las reacciones químicas. En cuanto a esto último según este estudio se muestra la disminución de la energía de activación (Westbrook \& Dryer, 1981).

Sin embargo, cuando se adicionan cantidades más altas de metano, $10 \%$ y $14 \%$, se observa un incremento considerable de la energía de activación, esto puede ser debido a la inclusión de una cantidad considerable de un combustible gaseoso, metano, donde las reacciones en fase homogénea (metano con oxígeno) son más rápidas comparadas con las de la fase heterogénea (oxígeno-carbonizado) tal y como se muestra en Esquema de reacción 1 (Warnatz, Maas, \& Dibble, 2006).

De acuerdo al Esquema de reacción 1, todo dependerá principalmente de sí el metano se encuentra en relación estequiométrica con el oxígeno para ser oxidado directamente,

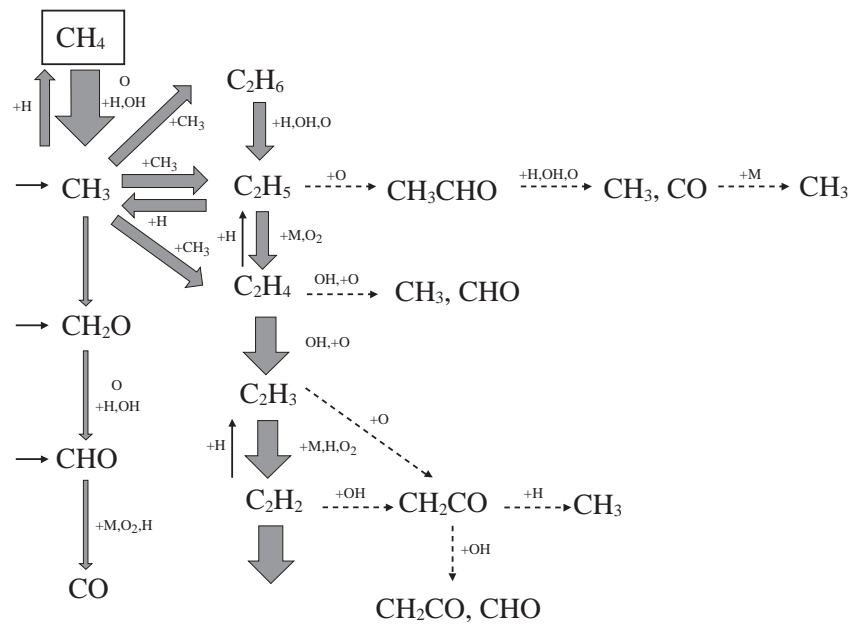

Esquema de reacción 1. Mecanismo de reacción del metano en una llama de mezcla rica (Warnatz, et al., 2006) 
pero en éste caso, la mezcla es rica en metano, y por lo tanto la ruta de oxidación implica en primer lugar recombinar los radicales producidos a partir del metano produciendo etano $\left(\mathrm{C}_{2} \mathrm{H}_{6}\right)$ el cual es posteriormente oxidado (Warnatz, et al., 2006). Por consiguiente, en concentraciones altas de metano se produce un efecto contraproducente ya que los radicales formados compiten con el carbón por los compuestos oxigenados disponibles.

\section{Conclusiones}

Se observó tendencia a disminuir la energía de activación cuando se aumentaba la concentración de oxígeno, logrando un valor de 90,69 kJ/mol cuando se empleó $21 \%$ de oxígeno y el modelo cinético de velocidad máxima $A$, valor que se asemeja a los reportados en la literatura con un buen factor de correlación $\mathrm{R}^{2}$. Al obtener los parámetros cinéticos para las mezclas de $\mathrm{O}_{2} / \mathrm{CH}_{4}$ con $5 \%$ de oxígeno y variando el metano a 5,10 y $14 \%$ con éste mismo método, se observó que para la mezcla $5 \%$ de cada gas el valor de la energía de activación disminuyó en un 10\% aproximadamente con un valor de 110,70 kJ/mol comparativamente con el valor obtenido para una atmósfera de reacción compuesta únicamente por oxígeno al $5 \%$ cuyo valor fue de $122,90 \mathrm{~kJ} / \mathrm{mol}$ evidenciando un efecto promotor del metano que produce radicales necesarios para mejorar la reacción de oxidación; al aumentar gradualmente la concentración de metano, se observó un aumento de la energía de activación obteniendo un valor de 170,80 $\mathrm{kJ} / \mathrm{mol}$ esto debido a las reacciones de oxidación competitivas entre el oxígeno con ambos combustibles presentes que evita que el carbonizado de carbón sea oxidado de manera más eficiente.

De acuerdo a lo anterior, se concluye finalmente que la mezcla $\mathrm{O}_{2} / \mathrm{CH}_{4}$ con $5 \%$ de cada gas presenta un efecto promotor en la oxidación del carbonizado de carbón, lo que podría conllevar a la disminución de la proporción de inquemados sólidos que se han convertido en un problema cuando se hace combustión bajo condiciones de combustión sin llama o MILD combustion.

\section{Agradecimientos}

Los autores agradecen al "Departamento Administrativo de Ciencia, Tecnología e Innovación”- COLCIENCIAS- a través del programa "Investigación e innovación en combustión avanzada en el uso industrial" por la financiación a través del proyecto "Desarrollo y evaluación de un quemador de $5 \mathrm{~kW}$ que opere en el régimen de combustión sin llama y que emplee como combustible carbón o una mezcla de este con gas natural" y al programa Sostenibilidad de la Universidad de Antioquia por el soporte financiero.

\section{Conflicto de intereses}

No se presenta conflicto de intereses.

\section{Referencias}

Acelas, N., Ruiz, W., López, D. (2010). Determinación de los parámetros cinéticos en la pirólisis del pino Ciprés. Quim. Nova, 33 (7): 1500-1505.
Alvarado, P., Cadavid, F., Mondragón, F., Ruiz, W., \& Amell, A. (2009). Estudio cinético químico de la formación de óxidos de nitrógeno en la combustión sin llama del metano. Energética, 41: 13-22.

Alviso, D., Rolon, J. C., Scouflaire, P., \& Darabiha, N. (2015). Experimental and numerical studies of biodiesel combustion mechanisms using a laminar counterflow spray premixed flame. Fuel, 153: 154-165. http://doi.org/10.1016/ j.fuel.2015.02.079

American Society of Testing and Materials. (1998). ASTM D338: Standard Classifiation of Coals by Rank (Vol. 552).

Bilgen, S. (2014). Structure and environmental impact of global energy consumption. Renewable and Sustainable Energy Reviews, 38: 890-902. http://doi.org/10.1016/j.rser.2014. 07.004

Cavaliere, A., \& De Joannon, M. (2004). Mild combustion. Mild Combustion (Vol. 30). Progress in Energy and Combustion Science. http://doi.org/10.1016/j.pecs.2004.02.003

Friedl, A., Padouvas, E., Rotter, H., \& Varmuza, K. (2005). Prediction of heating values of biomass fuel from elemental composition. Analytica Chimica Acta, 544 (1-2 SPEC. ISS.): 191-198. http://doi.org/10.1016/j.aca.2005.01.041

Gil, M. V., Casal, D., Pevida, C., Pis, J. J., \& Rubiera, F. (2010). Thermal behaviour and kinetics of coal/biomass blends during co-combustion. Bioresource Technology, 101 (14): 5601-5608. http://doi.org/10.1016/j.biortech.2010.02.008

Izquierdo, J. F., \& Torres, J. F. I. (2004). Cinética de las reacciones químicas. Cinética de las reacciones químicas. Barcelona: Universitat de Barcelona.

Jones, J. C., Chiz, P. S., Koh, R., \& Matthew, J. (1996). Kinetic parameters of oxidation of bituminous coals from heatrelease rate measurements. Fuel, 75 (15): 1755-1757. http:// doi.org/10.1016/S0016-2361(96)00159-7

Krerkkaiwan, S., Fushimi, C., Tsutsumi, A., \& Kuchonthara, P. (2013). Synergetic effect during co-pyrolysis/gasification of biomass and sub-bituminous coal. Fuel Processing Technology, 115: 11-18. http://doi.org/10.1016/j.fuproc. 2013.03.044

Li, J., Yang, W., Blasiak, W., \& Ponzio, A. (2012). Volumetric combustion of biomass for $\mathrm{CO} 2$ and NOx reduction in coalfired boilers. Fuel, 102: 624-633. http://doi.org/10.1016/j. fuel.2012.06.083

Li, S., \& Yue, C. (2003). Study of different kinetic models for oil shale pyrolysis, 85: 51-61. http://doi.org/10.1016/S03783820(03)00097-3

Liotta, R., Brons, G., Isaacs, J. (1983). Oxidative weathering of Illinois No. 6 coal, 62 (6): 781-791. http://doi.org/doi. org/10.1016/0016-2361(83)90028-5

Masel, R. I. (1996). Principles of adsorption and reaction on solid surfaces, 804.

Medwell, P. R., \& Chinnici, A. (2017). Effect of particle size on the MILD combustion characteristics of pulverised brown coal. Fuel Processing Technology, 155 (November 2016): 74-87. http://doi.org/10.1016/j.fuproc.2016.04.003

Park, D. K., Kim, S. D., Lee, S. H., \& Lee, J. G. (2010). Copyrolysis characteristics of sawdust and coal blend in TGA and a fixed bed reactor. Bioresource Technology, 101 (15): 6151-6156. http://doi.org/10.1016/j.biortech.2010.02.087

Perrin, N., Dubettier, R., Lockwood, F., Tranier, J.-P., BourhyWeber, C., \& Terrien, P. (2014). Oxycombustion for coal power plants: Advantages, solutions and projects. Applied Thermal Engineering, 74: 75-82. http://doi.org/10.1016/j. applthermaleng.2014.03.074 
Quan, C., Xu, S., An, Y., \& Liu, X. (2014). Co-pyrolysis of biomass and coal blend by TG and in a free fall reactor. Journal of Thermal Analysis and Calorimetry, 117 (2): $817-$ 823. http://doi.org/10.1007/s10973-014-3774-7

Saha, M., Chinnici, A., Dally, B. B., \& Medwell, P. R. (2015). Numerical Study of Pulverized Coal MILD Combustion in a Self- Recuperative Furnace. http://doi.org/10.1021/acs. energyfuels.5b01644

Shen, J., Liu, J., Ma, J., Zhang, H., \& Jiang, X. (2015). Parametric study of reburning of nitrogen oxide for superfine pulverized coal. Energy Conversion and Management, 89 (x): 825832. http://doi.org/10.1016/j.enconman.2014.10.059

Shi, L., Liu, Q., Guo, X., He, W., \& Liu, Z. (2014). Pyrolysis of coal in TGA: Extent of volatile condensation in crucible. Fuel Processing Technology, 121: 91-95. http://doi.org/10. 1016/j.fuproc.2014.01.013

Tjatjopoulos, G. J., \& Vasalos, I. a. (1992). Reaction-path analysis of a homogeneous methane oxidative coupling mechanism. Applied Catalysis A: General, 88 (2): 213-230. http://doi.org/10.1016/0926-860X(92)80216-Y

Torres Alvarado, P. N. (2014). Combustión de material carbonoso en condiciones de alta temperatura y baja concentración de oxígeno. Universidad de Antioquia (Tesis doctoral).
Wang, G., Zhang, J., Shao, J., Sun, H., \& Zuo, H. (2014). Thermogravimetric Analysis of Coal Char Combustion Kinetics. Journal of Iron and Steel Research, International, 21 (10): 897-904. http://doi.org/10.1016/S1006-706X(14)60159-X

Warnatz, J., Maas, U., \& Dibble, R. W. (2006). J. Warnatz · U. Maas $\cdot$ R.W. Dibble Combustion. New York.

Weber, R., Smart, J. P., \& Vd Kamp, W. (2005). On the (MILD) combustion of gaseous, liquid, and solid fuels in high temperature preheated air. Proceedings of the Combustion Institute, $30 \mathrm{II}(2)$ : 2623-2629. http://doi.org/10.1016/j.proci. 2004.08.101

Westbrook, C. K., \& Dryer, F. L. (1981). Simplified Reaction Mechanisms for the Oxidation of Hydrocarbon Fuels in Flames. Combustion Science and Technology, 27 (1-2): 3143. http://doi.org/10.1080/00102208108946970

Yuan, S., Dai, Z. H., Zhou, Z. J., Chen, X. L., Yu, G. S., \& Wang, F. C. (2012). Rapid co-pyrolysis of rice straw and a bituminous coal in a high-frequency furnace and gasification of the residual char. Bioresource Technology, 109: 188-197. http://doi.org/10.1016/j.biortech.2012.01.019 